\title{
ESTUDO DA VARIAÇÃO DA DBCP E DO ÂNGULO DE SOLDAGEM EM CORRENTE PULSADA, CURTO CIRCUITO CONVENCIONAL E CURTO CIRCUITO CONTROLADO EM SOLDAGEM MIG/MAG *
}

Jamil Nobre de Castro ${ }^{1}$ Bruno Ribeiro Pinheiro ${ }^{2}$ Marcelo Ferreira Motta ${ }^{3}$

\section{Resumo}

O objetivo desse trabalho é comparar três técnicas em soldagem MIG/MAG com baixa energia: Curto Circuito convencional, Curto Circuito Controlado (CCC) Soft e modo corrente Pulsada através de soldagens na posição vertical descendente e avaliando a geometria do cordão de solda, o acabamento superficial e os sinais de aquisição. Essas três técnicas tem como vantagem a soldagem em todas as posições e o uso de baixas energias, que otimizam seu uso em chapas muito finas e em de passes de raiz. Foram definidos alguns parâmetros constantes como velocidade de alimentação e velocidade de soldagem e a corrente média foi mantida aproximadamente igual. Os parâmetros trabalhados foram a Distância Bico de Contato a Peça (DBCP) e o ângulo de soldagem e foi utilizado o modelo experimental Fatorial Completo, resultando em 27 ensaios. A soldagem com CCC Soft manteve a qualidade em quase todas as soldagens enquanto 0 C.C convencional e a corrente pulsada tiveram resultados satisfatórios apenas em algumas condições. Os resultados apresentaram que as soldagens com CCC Soft mantiveram a a geometria e a energia aproximadamente iguais mesmo com a variação da DBCP e do ângulo, o que não aconteceu com as outras duas técnicas, e a corrente pulsada apresentou bons resultados apenas na posição 15 graus puxando.

Palavras-chave: MIG/MAG; Curto Circuito; CCC; Corrente pulsada.

\section{STUDY OF THE VARIATION OF THE CTWD AND WELDING ANGLE IN PULSED CURRENT, CONVENTIONAL SHORT CIRCUIT AND CONTROLLED SHORT CIRCUIT IN MIG / MAG WELDING}

\section{Abstract}

The objective of this work is to compare three techniques in low energy MIG / MAG welding: Conventional Short Circuit, Short Circuit Controlled (CCC) Soft and current mode Pulsed through welds in the downward vertical position and evaluating the weld bead geometry, the finish and acquisition signs. These three techniques have the advantage of welding in all positions and the use of low energies, which optimize their use in very thin plates and in root passes. Some constant parameters were defined as feed rate and welding speed and average current was maintained approximately equal. The parameters worked were the Contact Tip to Work Distance (CTWD) and the welding angle and the full factorial experiment model was used, resulting in 27 tests. CCC Soft welding maintained the quality in almost all welds while conventional short circuit and pulsed current had satisfactory results under some conditions only. The results showed that the welds with CCC Soft kept the geometry and the energy approximately equal even with the variation of the DBCP and the angle, which did not happen with the other two techniques, and the pulsed current presented good results only in the position 15 degrees pulling.

Keywords: MIG/MAG; Short circuit, CCC ;Pulsed Current.

Estudante de graduação em Engenharia Metalúrgica na Universidade Federal do Ceará, Brasil.

Estudante de graduação em Engenharia Metalúrgica na Universidade Federal do Ceará, Brasil.

Professor do departamento de Engenharia Metalúrgica e de Materiais na Universidade Federal do Ceará, Brasil. 


\section{INTRODUÇÃO}

Entre os processos de soldagem, o MIG (metal inert gas) e o MAG (metal active gás) são bastante versáteis e possibilitam a operação com muitos modos de transferência metálica, tais como globular, goticular (spray) e curto circuito e se destacam por garantirem uma alta produtividade e a possibilidade de robotização. No processo MIG/MAG, se for escolhido na fonte o modo tensão constante (conhecido como Convencional), para baixos valores de corrente média, tensão média e curta DBCP, a transferência metálica ocorre por curto circuito, no qual um arame-eletrodo continuamente alimentado é fundido e depositado durante períodos de $20-150 \mathrm{hz}$ formando uma gota que cresce e é sugada para a poça de fusão pela força da tensão superficial[1,2]. É importante mencionar que nesse tipo de transferência, o arco elétrico é apagado a cada curto circuito e reaberto após o destacamento da gota.

A desvantagem da transferência por curto circuito no modo convencional é de que a qualidade da solda tende a ser regular, com baixa estabilidade do arco e presença de muitos respingos. Para amenizar esse problema, foi desenvolvido o curto circuito controlado (CCC), que controla a transferência metálica através da imposição de uma forma de onda de corrente, que possibilita maior estabilidade na transferência de curto circuito, evitando a formação de respingos grosseiros e choques abruptos com a peça, aumentando a confiabilidade e produtividade do processo.

O Curto Circuito Controlado Soft usado no presente trabalho é uma tecnologia da IMC presente nas fontes de soldagem Digilpus A7. A figura 1 apresenta a forma de onda de corrente desta modalidade, onde em amarelo é representada o período de curto circuito. $\mathrm{O}$ controle da corrente é feito, tanto durante o curto, quanto durante 0 arco. Durante o curto a corrente irá aumentar na taxa definida por Irps, até que ocorra a reabertura do arco. Após a reabertura, a fonte irá aplicar a corrente la, descendo de acordo com taxa Irpd, até atingir o valor de corrente If. A corrente irá permanecer em If até ocorrer o curto circuito. Após o curto circuito a corrente poderá permanecer, ainda, em If durante o tempo de atraso ta. Nesta modalidade o tempo de atraso ta é relacionado com o assentamento da gota e la visa reduzir as perturbações no momento da reabertura, bem como, atuar sobre a energia fornecida ao arco [3].

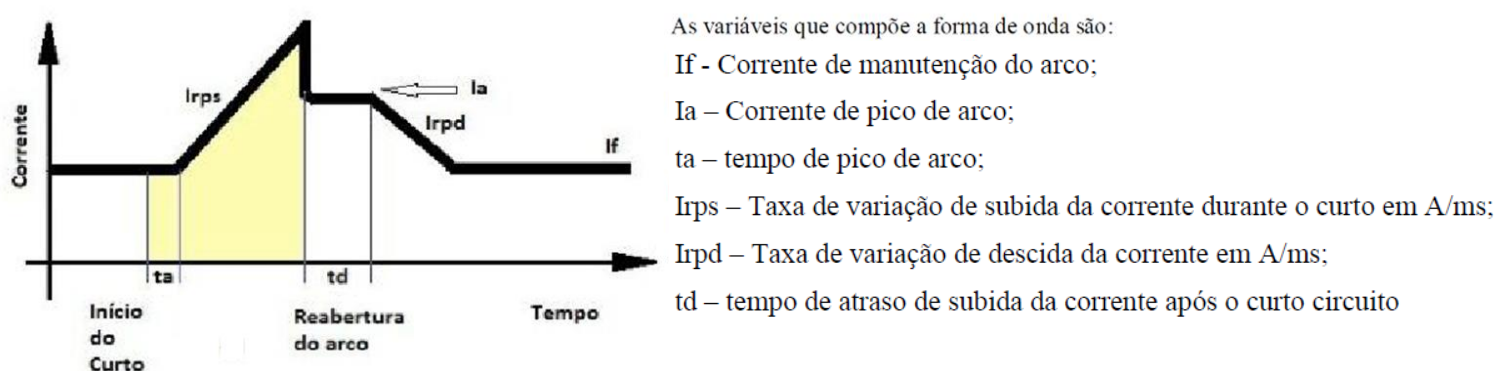

Figura 1: forma da corrente no Curto Circuito Controlado Soft [3]

MIG/MAG Pulsado é um modo de transferência controlado eletronicamente. Nesta variante do processo MIG/MAG, a corrente é também a variável de soldagem controlada, que passa a oscilar entre dois níveis, as chamadas correntes de base e de pulso. Busca-se o destacamento de uma gota por período de pulso de corrente, obtendo-se uma transferência do metal semelhante ao modo spray, porém, em 
baixos níveis de corrente média [2]. Esse modo de soldagem foi utilizado no trabalho porque é possível ter uma corrente média semelhante as transferencias por curto circuito

\subsection{Parâmetros trabalhados}

A inclinação da tocha ou ângulo de deslocamento da tocha (figura 2) é o ângulo entre o eixo do eletrodo e a linha normal ao eixo do cordão de solda. Quando o ângulo é positivo( técnica "puxando" ou seja , eletrodo apontando para o cordão já formado), o cordão de solda apresenta maior penetração, menos salpicos, é mais estreito, mais convexo e o arco é mais estável. Quando se utiliza o ângulo negativo (técnica "empurrando") estão presentes diversar características tais como menor penetração, mais salpicos, cordão mais plano e mais largo. Esta técnica é empregada quando grandes valores de penetração não são requiridos. Quando se utilizado o ângulo nulo $\left(0^{\circ}\right)$, tem-se características intermediárias dos dois casos citados anteriormentes [4].

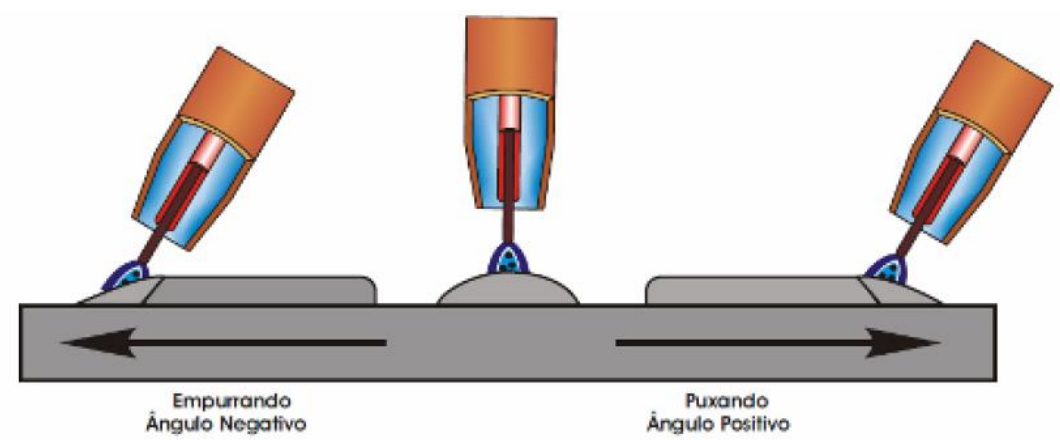

Figura 2: Ângulo de soldagem [5]

A Distância de Bico de Contato Peça (DBCP) é considerada uma variável de soldagem do processo MIG/MAG, a sua variação pode gerar alterações significativas tanto na corrente média quanto na freqüência de transferência metálica, principalmente no modo curto-circuito. Esta variação afeta tanto o comprimento do eletrodo ("stickout") quanto o comprimento de arco (figura 3), fatores estes que têm influência no consumo e na corrente, respectivamente [6].

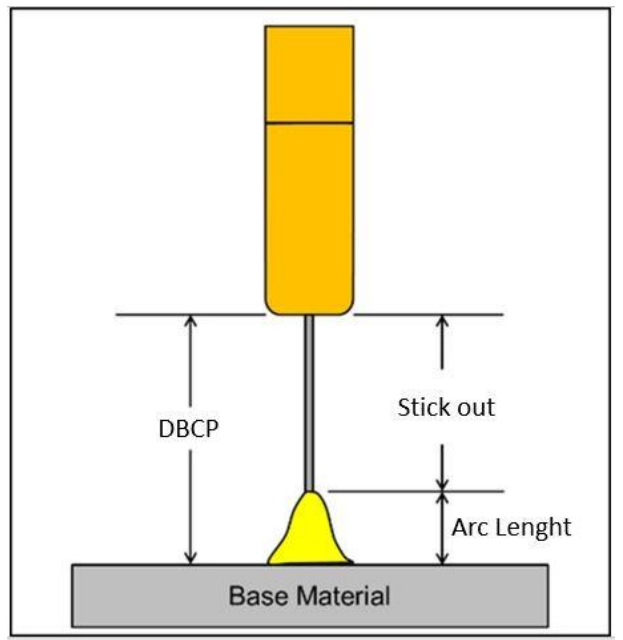

Figura 3: DBCP [7] 
Esse trabalho apresentado tem como objetivo realizar um estudo comparativo entre as formas de transferência metálica por curto circuito convencional e controlado e também com corrente pulsada em soldagens na posição vertical descendente.

\section{MATERIAIS E MÉTODOS}

As soldagens aconteceram no Laboratório de Tecnologia e Pesquisa em Soldagem (LPTS) localizado na Universidade Federal do Ceará (UFC) com o auxílio de uma fonte de soldagem IMC digiplus A7, um robô Kuka para a automação da solda e o programa para aquisição de dados SapV4. Foram realizadas simples deposições de um arame AWS ER 70S-6 de 1,2 mm de diâmetro sobre uma barra chata de 12,6 $\mathrm{mm}$ de espessura e grau ASTM A-36 (tabela 1).

Tabela 1. Composição química do arame e do metal base

\begin{tabular}{lccccc}
\hline Material & \%C & \%Si & $\%$ Mn & \%S & \%P \\
\hline $\begin{array}{l}\text { AWS ER } \\
70 S-6\end{array}$ & 0,15 & 1,15 & 1,85 & 0,035 & 0,025 \\
\hline ASTM A-36 & 0,27 & 0,4 & 0,90 & 0,04 & 0,05 \\
\hline
\end{tabular}

Foram definidos alguns parâmetros como fixos (tabela 2) e em seguida foram realizados ensaios exploratórios na posição plana para definição dos parâmetros antes de iniciar as soldagens na posição vertical. As velocidades de soldagem (Vs) e de alimentação do arame ( $\mathrm{Va}$ ) foram mantidas constante em todas as condições para assegurar que a quantidade de material depositada seja a mesma. O objetivo dos ensaios exploratórios é alcançar a mesma corrente média nas 3 técnicas com cordões de boa aparência.

\begin{tabular}{ccccc} 
Tabela 2. Parâmetros fixos \\
\hline Posição & $\begin{array}{c}\text { Vs } \\
\text { (m/min) }\end{array}$ & $\begin{array}{c}\text { Va } \\
(\mathbf{m} / \mathbf{m i n})\end{array}$ & $\begin{array}{c}\text { Gás de } \\
\text { proteção }\end{array}$ & $\begin{array}{c}\text { Vazão } \\
\text { (L/min) }\end{array}$ \\
\hline $\begin{array}{c}\text { Vertical } \\
\text { descendente }\end{array}$ & 0,25 & 3,5 & $4 \% \mathrm{CO}_{2}$ & 16 \\
\hline
\end{tabular}

\subsection{Ensaios exploratórios}

Para encontrar a melhor condição no curto circuito convencional foram realizadas quatro soldagens, com $17 \mathrm{~V}, 18 \mathrm{~V}, 19 \mathrm{~V}$ e $20 \mathrm{~V}$, e foi utilizado o aspecto visual e 0 Índice Vilarinho de Regularidade da Transferência por Curto-Circuito cujo símbolo é Ivcc para avaliar a melhor condição. Segundo Vilarinho [8], a regularidade de uma transferência pode ser estimada através dos desvios padrões das médias do tempo de curto circuito e de arco aberto e das médias do tempo de curto circuito e de arco aberto (Equação 1). Os valores de indutância $\mathrm{Vs}$ e $\mathrm{Vd}$ foram mantidos constantes em $100(\mathrm{~A} / \mathrm{ms})$ e $60(\mathrm{~A} / \mathrm{ms})$ respectivamente. Alteração nesses valores influenciam no tempo de arco aberto e de curto circuito e um valor inadequado pode causar instabilidade na transferência metálica.

$$
\mathrm{IV}_{\mathrm{cc}}=\frac{\sigma_{\mathrm{tcc}}}{\mathrm{t}_{\mathrm{cc}}}+\frac{\sigma_{\mathrm{tab}}}{\mathrm{t}_{\mathrm{ab}}}
$$

Equação 1. Índice vilarinho de estabilidade, onde quanto menor o IVcc mais estável tende a ser a soldagem. [8] 
O CCC Soft possui seis parâmetros de operação e para reduzir o número de soldagens exploratórias as taxas de variação da corrente na subida (Irps) e na descida (Irpd) foram mantidas constantes em 100 (A/ms) e 40 (A/ms) respectivamente, sobrando 4 parâmetros para serem trabalhados. Para obtenção da melhor condição foi utilizado o modelo de experimentos Taguchi [9] na categoria "quanto menor melhor" com uma matriz formulada para 4 fatores e 3 níveis (tabela 3) resultando em 9 ensaios (tabela 4). A técnica desenvolvida por Taguchi é uma ferramenta poderosa na otimização de produtos ou processo e visa determinar as variáveis que mais influenciam a "variabilidade" do processo ou produto. O controle destes fatores garante a qualidade do processo/produto além da otimização do seu desempenho [10]. A técnica estatística mais empregada para interpretação dos dados experimentais é a ANOVA (análise de variância). Também foi utilizado o índice IVcc de qualidade.

Tabela 3. Fatores e niveis dos parâmetros

\begin{tabular}{cccc}
\hline Fatores & \multicolumn{3}{c}{ Niveis } \\
\hline & $\mathbf{1}$ & $\mathbf{2}$ & $\mathbf{3}$ \\
\hline If & 80 & 100 & 120 \\
\hline la & 190 & 220 & 250 \\
\hline $\mathrm{Ta}$ & 1 & 1,5 & 2 \\
\hline $\mathrm{Td}$ & 1 & 1,5 & 2 \\
\hline${ }^{*}$ Cada fator possui 3 niveis &
\end{tabular}

\begin{tabular}{ccccc}
\multicolumn{6}{l}{ Tabela 4. Matriz ortogonal L9 com as combinações } \\
\hline Experimento & If & la & Ta & Td \\
\hline 1 & 1 & 1 & 1 & 1 \\
\hline 2 & 1 & 2 & 2 & 2 \\
\hline 3 & 1 & 3 & 3 & 3 \\
\hline 4 & 2 & 1 & 2 & 3 \\
\hline 5 & 2 & 2 & 3 & 1 \\
\hline 6 & 2 & 3 & 1 & 2 \\
\hline 7 & 3 & 1 & 3 & 2 \\
\hline 8 & 3 & 2 & 1 & 3 \\
\hline 9 & 3 & 3 & 2 & 1 \\
\hline
\end{tabular}

Matriz de experimentos com os niveis de cada fator

Os ensaios exploratórios em corrente pulsada ocorreram após as duas formas de curto circuito, então já se havia o conhecimento de qual corrente média estava buscando. Foi usada a equação 2 para estimar a corrente média .

$$
I_{m}=\left(I_{b \times} t_{b}+I_{p \times} t_{p}\right) \div\left(t_{b}+t_{p}\right)
$$

Equação 2: Cálculo da corrente média em corrente pulsada[11]

\subsection{Soldagens definitivas}

Foi montado um modelo de experimentos do tipo fatorial completo (tabela 5), onde todas as combinações entre DBCP, ângulo e técnica são testadas, resultando em 27 ensaios.

Tabela 5. Fatorial completo

\begin{tabular}{cccc}
\hline № & Técnica & DBCP & Ângulo \\
\hline 1 & Convencional & 20 & $0^{\circ}$ \\
\hline 2 & Convencional & 17 & $0^{\circ}$ \\
\hline 3 & Convencional & 14 & $0^{\circ}$ \\
\hline 4 & CCC Soft & 20 & $0^{\circ}$ \\
\hline 5 & CCC Soft & 17 & $0^{\circ}$ \\
\hline 6 & CCC Soft & 14 & $0^{\circ}$ \\
\hline 7 & Pulsada & 20 & $0^{\circ}$ \\
\hline 8 & Pulsada & 17 & $0^{\circ}$ \\
\hline 9 & Pulsada & 14 & $0^{\circ}$ \\
\hline 10 & Convencional & 20 & $+15^{\circ}$ \\
\hline
\end{tabular}




\begin{tabular}{rccc}
\hline 11 & Convencional & 17 & $+15^{\circ}$ \\
\hline 12 & Convencional & 14 & $+15^{\circ}$ \\
\hline 13 & CCC Soft & 20 & $+15^{\circ}$ \\
\hline 14 & CCC Soft & 17 & $+15^{\circ}$ \\
\hline 15 & CCC Soft & 14 & $+15^{\circ}$ \\
\hline 16 & Pulsada & 20 & $+15^{\circ}$ \\
\hline 17 & Pulsada & 17 & $+15^{\circ}$ \\
\hline 18 & Pulsada & 14 & $+15^{\circ}$ \\
\hline 19 & Convencional & 20 & $-15^{\circ}$ \\
\hline 20 & Convencional & 17 & $-15^{\circ}$ \\
\hline 21 & Convencional & 14 & $-15^{\circ}$ \\
\hline 22 & CCC Soft & 20 & $-15^{\circ}$ \\
\hline 23 & CCC Soft & 17 & $-15^{\circ}$ \\
\hline 24 & CCC Soft & 14 & $-15^{\circ}$ \\
\hline 25 & Pulsada & 20 & $-15^{\circ}$ \\
\hline 26 & Pulsada & 17 & $-15^{\circ}$ \\
\hline 27 & Pulsada & 14 & $-15^{\circ}$ \\
\hline Disposição dos ensaios segundo o modelo Fatorial Completo
\end{tabular}

\section{RESULTADOS E DISCUSSÃO}

\subsection{Resultados dos ensaios exploratórios}

Os melhores parâmetros que foram encontrados nos ensaios exploratórios e que serão utilizados nas soldagens definitivas estão nas tabelas 6, 7 e 8.

Tabela 6. Parâmetros para curto circuito Convencional

\begin{tabular}{|c|c|c|c|c|c|c|}
\hline $\begin{array}{l}\text { DBCP } \\
\text { (mm) }\end{array}$ & $\begin{array}{l}\text { V ref } \\
\text { (V) }\end{array}$ & $\begin{array}{c}\mathrm{Ks} \\
(\mathrm{A} / \mathrm{ms})\end{array}$ & $\begin{array}{c}\mathrm{Kd} \\
(\mathrm{A} / \mathrm{ms})\end{array}$ & $\begin{array}{l}\mathrm{Vm} \\
\text { (V) }\end{array}$ & $\begin{array}{l}\text { Im } \\
\text { (A) }\end{array}$ & $\begin{array}{l}\text { Energia } \\
(\mathrm{Kj} / \mathrm{mm})\end{array}$ \\
\hline 20 & 19 & 100 & 60 & 19,3 & 126 & 0,58 \\
\hline
\end{tabular}

Tabela 7. Parâmetros para Curto Circuito Controlado Soft

\begin{tabular}{cccccccccc}
\hline DBCP & $\begin{array}{c}\text { If } \\
(\mathbf{m m})\end{array}$ & $\begin{array}{c}\text { (A) } \\
\text { (A) }\end{array}$ & $\begin{array}{c}\text { Ta } \\
(\mathbf{m s})\end{array}$ & $\begin{array}{c}\text { Td } \\
(\mathbf{m s})\end{array}$ & $\begin{array}{c}\text { Irps } \\
(\mathbf{A} / \mathbf{m s})\end{array}$ & $\begin{array}{c}\text { Irpd } \\
(\mathbf{A} / \mathbf{m s})\end{array}$ & $\begin{array}{c}\text { Vm } \\
(\mathbf{V})\end{array}$ & $\begin{array}{c}\text { Im } \\
(\mathbf{A})\end{array}$ & $\begin{array}{c}\text { Energia } \\
(\mathrm{Kj} / \mathbf{m m})\end{array}$ \\
\hline 20 & 100 & 250 & 2 & 2 & 100 & 40 & 22,4 & 125 & 0,67 \\
\hline
\end{tabular}

Tabela 8. Parâmetros para o modo corrente Pulsada

\begin{tabular}{cccccccc}
\hline $\begin{array}{c}\text { (DBCP) } \\
(\mathbf{m m})\end{array}$ & $\begin{array}{c}\mathbf{I p} \\
(\mathbf{A})\end{array}$ & $\begin{array}{c}\mathrm{Tp} \\
(\mathbf{m s})\end{array}$ & $\begin{array}{c}\mathbf{I b} \\
(\mathbf{A})\end{array}$ & $\begin{array}{c}\mathrm{Tb} \\
(\mathbf{m s})\end{array}$ & $\begin{array}{c}\mathbf{V m} \\
(\mathbf{V})\end{array}$ & $\begin{array}{c}\mathbf{I m} \\
(\mathbf{A})\end{array}$ & $\begin{array}{c}\text { Energia } \\
(\mathbf{K j} / \mathbf{m m})\end{array}$ \\
\hline 20 & 330 & 2,2 & 60 & 6 & 28,9 & 129 & 0,89 \\
\hline
\end{tabular}

Nos ensaios exploratórios com o CCC Soft, após as nove soldagens, os resultados foram processados no software Statistica e os resultados se encontram na figura 4. Segundo o gráfico, quanto mais acima está o ponto, mais influente é aquele nível na redução do Ivcc. Segundo esse critério o nível 2 de If, 3 de la, 3 de Ta e 3 de Td (Tabela 7) são a melhor condição apontada pelo método. 


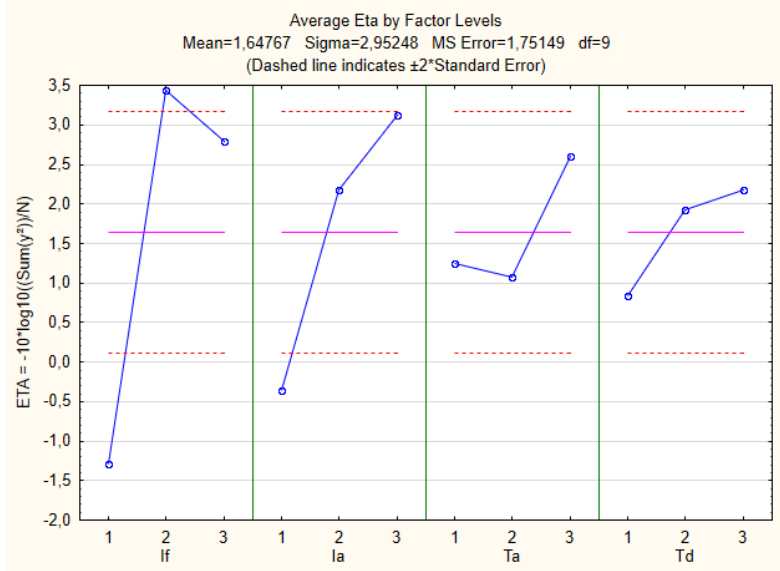

Figura 4: Melhor condição apontada pelo método taguchi (Software Statistica)

\subsection{Resultado das soldagens definitivas}

Após o posicionamento das barras chatas na posição vertical, as soldagens definitivas aconteceram em três etapas, na primeira, o ângulo da tocha foi definido em $0^{\circ}$, na segunda etapa em +15 graus e na terceira etapa em -15․ Em cada etapa foram realizadas as nove soldagens com todas as combinações de técnica e DBCP apresentadas na tabela $5 \mathrm{com}$ a metodologia fatorial completo. Nas três etapas, cada técnica foi soldada na mesma barra chata, sendo que a DBCP na mesma barra chata da direita para esquerda tem os respectivos valores de $20 \mathrm{~mm}, 17 \mathrm{~mm}$ e $14 \mathrm{~mm}$. Nas imagens 1, 2 e 3 é apresentado o curto circuito convencional. Os números acima do cordão de solda correspondem a ordem de soldagem de acordo com a tabela 5 e abaixo dos cordões estão uma amostra da macrografia do corte transversal..
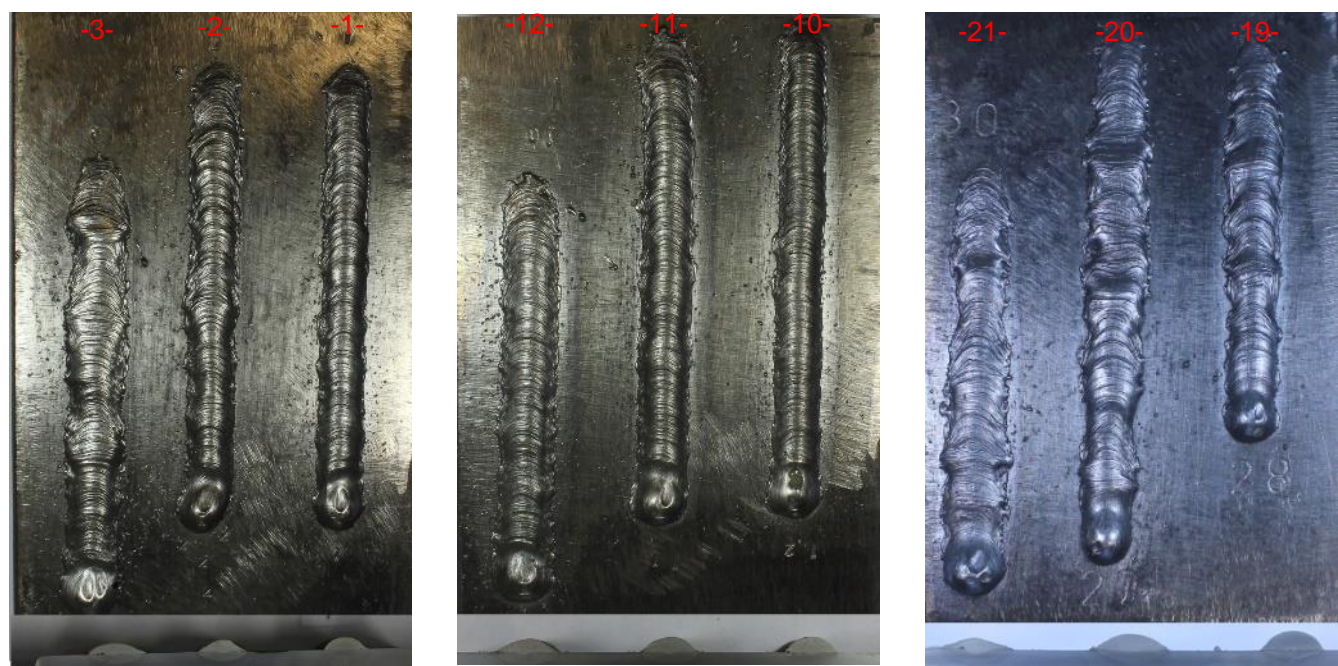

Imagens 1, 2 e 3: Curto circuito convencional. $0^{\circ}, 1^{\circ}$ e e -15을 respectivamente.

Nas imagens 4, 5 e 6 é apresentado os cordões de solda com transferência por CCC Soft. 
Todos os cordões de soldas foram cortados na serra fita e foram realizadas duas metalografias para cada soldagem em regiões centrais da solda.

Tabela 9. Todas as soldagens

\begin{tabular}{|c|c|c|c|c|c|c|c|c|c|}
\hline № & Técnica & DBCP & Ângulo & $\begin{array}{l}\operatorname{Im} \\
(\mathrm{A})\end{array}$ & $\begin{array}{l}\mathrm{Vm} \\
\text { (V) }\end{array}$ & $\begin{array}{l}\text { Energia } \\
(\mathrm{Kj} / \mathrm{mm})\end{array}$ & $\begin{array}{c}\text { Largura* } \\
\text { (mm) }\end{array}$ & $\begin{array}{c}\text { Penetração* } \\
(\mathbf{m m})\end{array}$ & $\begin{array}{c}\text { Reforço* } \\
(\mathrm{mm})\end{array}$ \\
\hline 1 & Convencional & 20 & $0^{\circ}$ & 126 & 19,3 & 0,58 & 8,85 & 0,20 & 2,41 \\
\hline 2 & Convencional & 17 & $0^{\circ}$ & 131 & 19,5 & 0,61 & 9,76 & 0,31 & 2,35 \\
\hline 3 & Convencional & 14 & $0^{\circ}$ & 137 & 19,4 & 0,64 & 10,19 & 0,32 & 1,99 \\
\hline 4 & CCC Soft & 20 & $0^{\circ}$ & 125 & 22,4 & 0,67 & 9,38 & 0,19 & 2,12 \\
\hline 5 & CCC Soft & 17 & $0^{\circ}$ & 127 & 22,5 & 0,69 & 9,09 & 0,30 & 2,14 \\
\hline 6 & CCC Soft & 14 & $0^{\circ}$ & 134 & 21,5 & 0,69 & 8,95 & 0,35 & 2,15 \\
\hline 7 & Pulsada & 20 & $0^{\circ}$ & 129 & 28,9 & 0,89 & 8,75 & 0,14 & 1,46 \\
\hline 8 & Pulsada & 17 & $0^{\circ}$ & 131 & 25,8 & 0,81 & 9,75 & 0,36 & 1,44 \\
\hline 9 & Pulsada & 14 & $0^{\circ}$ & 133 & 24,4 & 0,78 & 9,93 & 0,49 & 1,22 \\
\hline 10 & Convencional & 20 & $+15^{\circ}$ & 123 & 19,2 & 0,57 & 8,48 & 0,28 & 2,53 \\
\hline 11 & Convencional & 17 & $+15^{\circ}$ & 130 & 19,4 & 0,60 & 9,55 & 0,36 & 2,43 \\
\hline 12 & Convencional & 14 & $+15^{\circ}$ & 133 & 19,5 & 0,62 & 9,66 & 0,41 & 2,16 \\
\hline 13 & CCC Soft & 20 & $+15^{\circ}$ & 125 & 22,7 & 0,68 & 9,36 & 0,00 & 2,16 \\
\hline 14 & CCC Soft & 17 & $+15^{\circ}$ & 128 & 22,1 & 0,68 & 8,96 & 0,26 & 2,14 \\
\hline 15 & CCC Soft & 14 & $+15^{\circ}$ & 133 & 21,7 & 0,69 & 9,05 & 0,31 & 2,13 \\
\hline 16 & Pulsada & 20 & $+15^{\circ}$ & 129 & 27,3 & 0,85 & 9,81 & 0,12 & 2,13 \\
\hline 17 & Pulsada & 17 & $+15^{\circ}$ & 132 & 25 & 0,79 & 10,24 & 0,11 & 1,66 \\
\hline 18 & Pulsada & 14 & $+15^{\circ}$ & 134 & 25 & 0,80 & 10,79 & 0,31 & 1,65 \\
\hline 19 & Convencional & 20 & $-15^{\circ}$ & 128 & 19,3 & 0,59 & 9,04 & 0,06 & 2,51 \\
\hline 20 & Convencional & 17 & $-15^{0}$ & 133 & 19,3 & 0,61 & 9,98 & 0,13 & 2,23 \\
\hline 21 & Convencional & 14 & $-15^{0}$ & 138 & 19,3 & 0,64 & 10,30 & 0,31 & 1,46 \\
\hline 22 & CCC Soft & 20 & $-15^{0}$ & 126 & 22 & 0,66 & 8,81 & 0,40 & 2,17 \\
\hline 23 & CCC Soft & 17 & $-15^{0}$ & 131 & 21,4 & 0,67 & 8,62 & 0,38 & 2,24 \\
\hline 24 & CCC Soft & 14 & $-15^{0}$ & 137 & 21,2 & 0,70 & 9,07 & 0,43 & 2,27 \\
\hline 25 & Pulsada & 20 & $-15^{0}$ & 129 & 28,8 & 0,89 & 7,66 & 0,00 & 1,06 \\
\hline 26 & Pulsada & 17 & $-15^{0}$ & 130 & 26,7 & 0,83 & 9,51 & 0,24 & 1,39 \\
\hline 27 & Pulsada & 14 & $-15^{0}$ & 132 & 24,8 & 0,79 & 10,07 & 0,31 & 1,28 \\
\hline
\end{tabular}

\subsection{Influência do tipo de transferência metálica, da DBCP e do ângulo de soldagem nas características geométricas}

Com os resultados da macrografia foi analisado os efeitos dos fatores (tipos de transferência, DBCP e ângulo de soldagem) na geometria da solda (largura, reforço e penetração). A geometria foi analisada apenas nas duas modalidades de curto circuito, visto que na corrente pulsada o cordão de solda foi bastante irregular e com variações significativas de reforço e largura ao longo do cordão de solda.

\subsubsection{Largura}

No curto circuito convencional, o aumento da largura do cordão foi atribuído a diminuição da DBCP e a diminuição do ângulo de soldagem (condição empurrando). Enquanto que no CCC Soft as mudanças na DBCP e no ângulo não causaram alterações relevantes. 


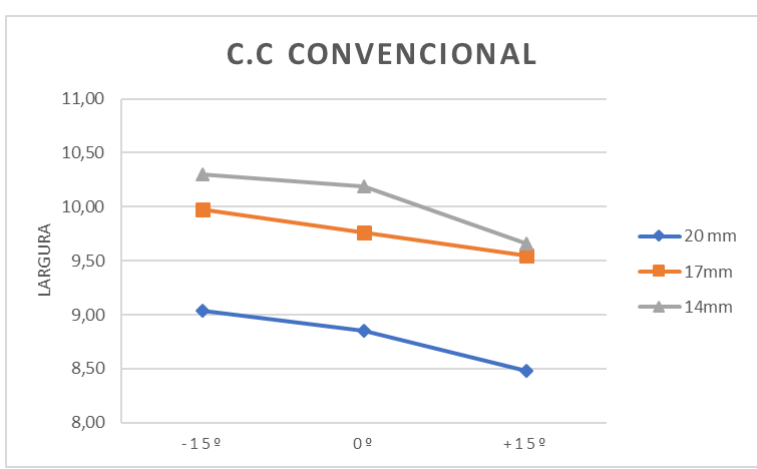

Figura 5. Largura x ângulo

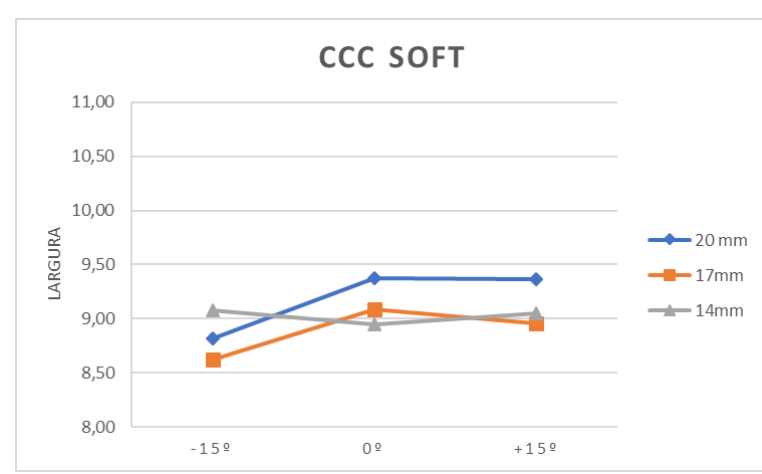

Figura 6. Largura $x$ ângulo

\subsubsection{Reforço}

A soldagem com CCC Soft não apresentou variação significativa do reforço com a mudança na DBCP e do ângulo enquanto no curto circuito convencional o reforço aumentou com a DBCP e diminuiu com a redução do ângulo de soldagem.

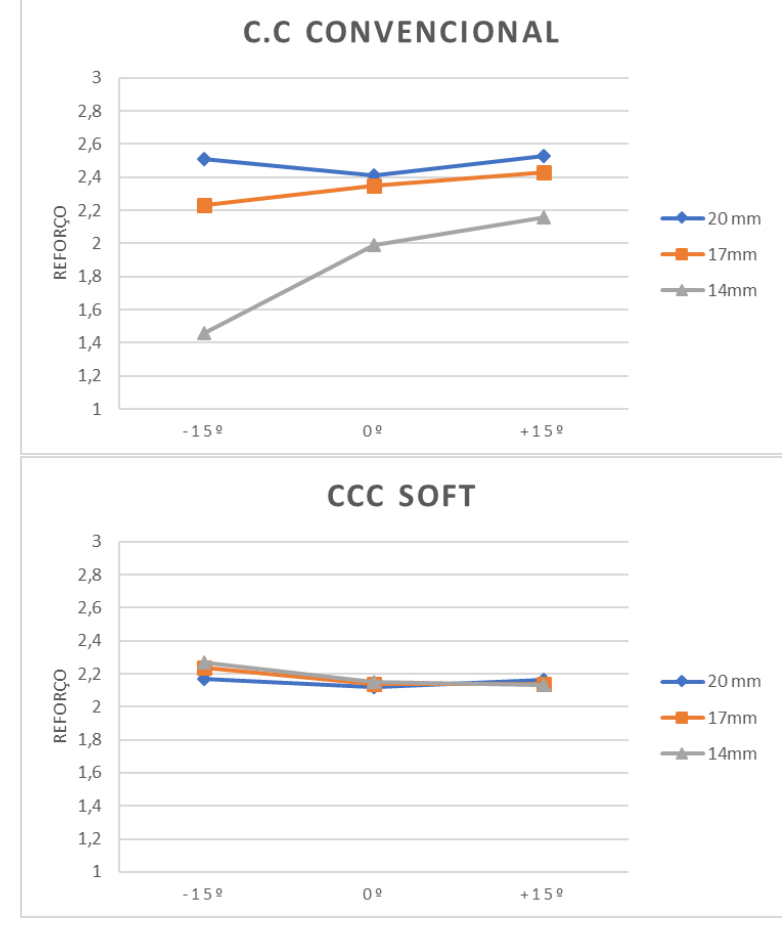

Figura 7. Reforço x ângulo
Figura 8. Reforço x ângulo

\subsubsection{Penetração}

A penetração aumentou com o aumento da DBCP nas duas formas de curto circuito e aumentou com o aumento do ângulo no c.c convencional enquanto diminuiu com o aumento do ângulo no CCC Soft. O valor de penetração zero na figura 10 foi o cordão de solda com incidêndia de poros, apresentado na condição 13 da imagem 5. 


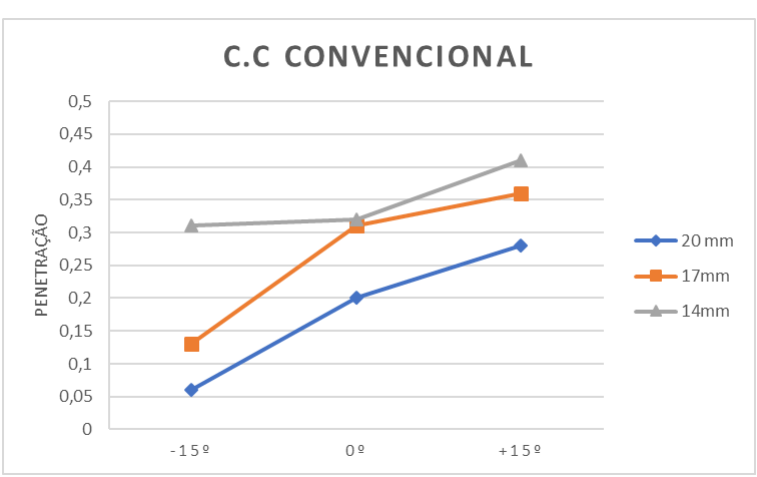

Figura 9. Penetração x ângulo

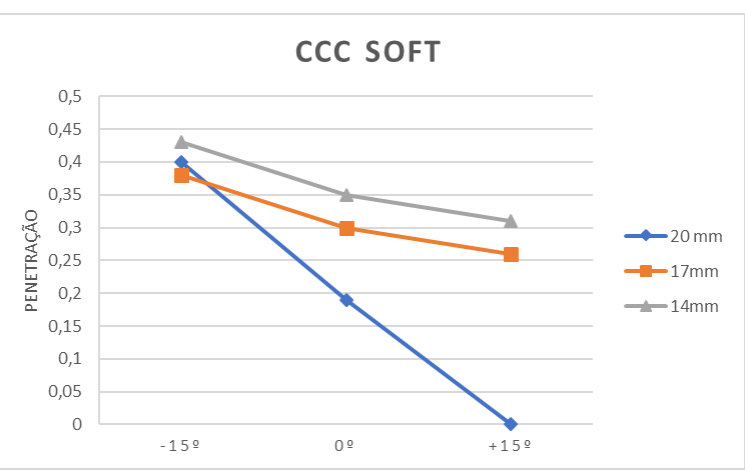

Figura 10. Penetração x ângulo

\section{CONCLUSÕES}

1. A DBCP se mostrou influente no curto circuito convencional, onde ocorreu a diminuição da energia de soldagem com o aumento da DBCP e na corrente pulsada, onde a energia de soldagem aumentou com o aumento da DBCP. No Curto Circuito Controlado SOFT o aumento da DBCP provocou pouca variação na energia, na largura e no reforço, mostrando-se um processo mais estável que o curto circuito convencional.

2. A corrente média de soldagem aumentou com a diminuição da DBCP nas três técnicas usadas e diminuiu com o aumento do ângulo de soldagem nas duas formas de curto circuito. Os curtos circuitos na DBCP de $14 \mathrm{~mm}$ e $17 \mathrm{~mm}$ fizeram a corrente média subir no modo corrente pulsada. Como a corrente pulsada não apresentou curto circuitos na DBCP de $20 \mathrm{~mm}$, a corrente de soldagem se manteve em 129 A nos três ângulos.

3. Verificou-se que a posição "empurrando" (ângulo negativo) produziu cordões de maior largura nas três técnicas, porém com algumas deformações na largura e reforço variando ao longo da solda.

4. A posição "puxando" (ângulo positivo) proporcionou as melhores soldas na técnica de corrente Pulsada e teve em $20 \mathrm{~mm}$ de DBCP e ângulo de $15^{\circ}$ a melhor condição (imagem 8).

5. Os resultados apontam que uma vez escolhido os parâmetros na técnica CCC Soft, a solda manterá a qualidade e terá mais mais confiabililidade do que na técnica curto circuito convencional.

6. Provou-se que a soldagem com corrente pulsada pode ser usada na posição vertical descendente desde que seja escolhido corretamento o ângulo e a DBCP.

\section{Agradecimentos}

Agradeço ao Programa de Educação Tutorial (PET) do curso de Engenharia Metalúrgica da UFC e ao Laboratório de Pesquisa em Tecnologia de Soldagem (LPTS) pela oportunidade de realizar esse trabalho.

\section{REFERÊNCIAS}

1 WAINER, E., BRANDI, S. D., \& MELLO, F. D. Soldagem: Processos e

Metalurgia. 1ํEd, São paulo: Editora Edgar Blucher,1992. p 99. 
FOGLIATTO, A.A.B. Influência dos parâmetros do processo MIG/MAG com Curto Circuito Controlado sobre a geometria do cordão de solda. Porto Alegre:

Universidade Federal do Rio Grande do Sul. Dissertação de Mestrado, 2013. p 1, 6.

MANUAL DE INSTRUÇÕES DIGIPLUS A7. Disponível em: < http://www.imcsoldagem.com.br/media/com_eshop/attachments/manual_Digiplus\%20AC_8aE d.pdf> Acesso em 20/05/2017.

4 GOLOB, F. Efeitos da mudança de Parâmetros na soldagem MAG CCC. Porto Alegre: Monografia, 2013. p 4.

5 SENAI-SP. Apostila soldagem: MIG/MAG básico. São paulo:2004. p 48

6 FERREIRA, F.F. Influência do tipo de gás de proteção da soldagem $\mathrm{mig} / \mathrm{mag}$ na qualidade do cordão de aço inoxidável. Disponível em: < http://www.grima.ufsc.br/cobef4/files/021008077.pdf>. Acesso em 10/05/2017.

7 PURSLOW, M. A Green Perspective on Arc Welding Part 1: Harnessing the Power of Resistive Heating. Disponível em: < https://ewi.org/a-green-perspective-on-arcwelding-part-1-harnessing-the-power-of-resistive-heating/> Acessado em 10/06/2017

8 VILARINHO. L.O. Avaliação de uma metodologia para seleção de parâmetros em soldagem MIG/MAG por curto circuito. CONEM 2010. Disponível em < http://www.abcm.org.br/anais/conem/2010/PDF/CON10-2367.pdf>. Acesso em 04/06/2017

9 ROSS, P,J. “Aplicação das Técnicas Taguchi na Engenharia da Qualidade”. 1991

10 MIRANDA, E.C. Estudo exploratório de parâmetros de soldagem de revestimento com ligas de níquel empregando o processo TIG com alimentação de arame frio. Fortaleza: Universidade Federal do Ceará. Dissertação de mestrado, 2009. p 33.

11 ANDRADE, A.F.C. Influência da corrente de pulso, tempo de pulso diâmetro da gota sobre a estabilidade da transferência metálica no processo MIG-P. Natal: Universidade Federal do Rio Grande do Norte.Dissertação de Mestrado,2012. p 35. 